\title{
Stress corrosion cracking of AISI 321 stainless steel in acidic chloride solution
}

\author{
YANLIANG HUANG \\ Marine Corrosion and Protection Laboratory, Institute of Oceanology, Chinese Academy of Sciences, 7 Nanhai Road, \\ Qingdao 266071, China
}

MS received 21 August 2001; revised 21 November 2001

\begin{abstract}
The stress corrosion cracking (SCC) of AISI 321 stainless steel in acidic chloride solution was studied by slow strain rate (SSR) technique and fracture mechanics method. The fractured surface was characterized by cleavage fracture. In order to clarify the SCC mechanism, the effects of inhibitor KI on SCC behaviour were also included in this paper. A study showed that the inhibition effects of KI on SCC were mainly attributed to the anodic reaction of the corrosion process. The results of strain distribution in front of the crack tip of the fatigue pre-cracked plate specimens in air, in the blank solution (acidic chloride solution without inhibitor KI) and in the solution added with KI measured by speckle interferometry (SPI) support the unified mechanism of SCC and corrosion fatigue cracking (CFC).
\end{abstract}

Keywords. Stress corrosion cracking; chloride; stainless steel; inhibitor.

\section{Introduction}

Stress corrosion cracking (SCC) is one of the most important causes of failure of stainless steel equipment and facilities (Collins and Monack 1973). Studies on the seashore facilities showed that an acidic chloride solution film formed on the surface of steel and the stainless steel in this media was in active dissolution state (Gnanamoorthy 1990; Kain 1990). It is generally thought that SCC cannot occur when the material is in active anodic dissolution state, but it occurred and some casualties (Oldfield and Todd 1990) were caused and this aroused the attention to the study of SCC. A number of mechanisms concerning SCC of materials have been proposed (Huang 1988; Newman and Procter 1990), but they cannot be used adequately to explain this kind of SCC. Some papers were devoted to the inhibition of SCC in stainless steels in acidic chloride-containing solutions (Chen and Wu 1985; Cao et al 1991). Chen and Wu (1985) studied the inhibition of a series of inorganic and organic additives on the SCC and pitting corrosion of AISI 321 stainless steel in an acidic chloride solution of $\mathrm{pH} 2.6$ at $90^{\circ} \mathrm{C}$ using U-bend specimens and it was concluded that the inhibition effects were attributed to the synergistic effect between organic amines and halide ions. Cao et al (1991) studied some organic and inorganic compounds in the inhibition of SCC of AISI 321 stainless steel in acidic chloride solution and found that among the effective inhibitors, iodides were the most effective ones. In this paper, the study on SCC mechanism and SCC inhibition were organically integrated. The study of inhibitor on anodic dissolution and deformation at crack tip provided some evidence of possible SCC mechanism. This also facilitated the selection of SCC inhibitors on this kind of SCC. The application of speckle interferometry (SPI) (Suezou and Hiroyosi 1985) in the in situ measurement of specimen in corrosion media was developed recently and it was found that this technique was suitable for the study of environment sensitive fracture of materials. A more detailed investigation into the role of $I^{-}$ in the inhibition of the SCC of AISI 321 stainless steel was then carried out by using SPI as well as conventional techniques and the results are presented here.

\section{Experimental}

\subsection{Materials and specimens}

The materials used for plate and rod specimens were both AISI 321 austenitic stainless steels. The specimen for the measurements of polarization curves was a rod $10 \mathrm{~mm}$ in diameter. The working surface was the cross-section. The rod specimen was used in slow strain rate (SSR) tests to determine SCC sensitivity and the effects of inhibitor KI on SCC. The single-edge pre-cracked plate specimens were used for the measurement of SCC crack propagation and strain distribution in front of the crack tip. The plate specimens were cut from $3 \mathrm{~mm}$ thick plate, parallel to the rolling direction. All the specimens were heat treated at $1050^{\circ} \mathrm{C}$ for $30 \mathrm{~min}$ in a salt bath and then quenched in water. When the specimens were treated as such, the microstructure of the specimens was single phase austenite. After heat treatment, the specimens were pickled for 
5 min in a $5 \% \quad \mathrm{HF}+20 \% \quad \mathrm{HNO}_{3}$ solution at room temperature, rinsed in distilled water thoroughly, followed by rinsing in acetone and then dried. For the rod specimens, they were coated with polytetrafluoroethylene except for the $20 \mathrm{~mm}$ working section that was totally immersed in the test solution.

The plate specimens were fatigue pre-cracked in air $\sim 1.5 \mathrm{~mm}$ beyond the notch tip. The plate was thin enough so that plain stress conditions could be assumed. The stress intensity factor $K$ at the crack tip can be calculated from the following formula (Chinese Research Institute of Aeronautical Technology 1981),

$$
\begin{gathered}
K=F \sigma \sqrt{\pi a} \\
F=\sqrt{\frac{2 b}{\pi a} \operatorname{tg} \frac{\pi a}{2 b}} \frac{0 \cdot 752+2 \cdot 02\left[\frac{a}{b}\right]+0 \cdot 37\left[1-\sin \frac{\pi a}{2 b}\right]^{3}}{\cos \frac{\pi a}{2 b}},
\end{gathered}
$$

where $a$ is the crack length, $b$ the plate width, $\sigma$ the stress.

\subsection{Test solution}

It was known from previous work (Cao et al 1991) that AISI 321 stainless steel is sensitive to SCC in a solution of $0.5 \mathrm{~mol} / 1 \mathrm{NaCl}+0.5 \mathrm{~mol} / 1 \mathrm{HCl}$ (blank solution) at $55^{\circ} \mathrm{C}$, so all tests were performed under this condition. The blank solution was prepared with pure reagent grade chemicals and distilled water. KI with pure reagent grade was used as the additive. The concentration of KI was $0.005 \mathrm{~mol} / \mathrm{l}$.

\subsection{Apparatus and procedures}

The SCC tests were carried out on a small SSR tensile test machine. The crack propagation of plate specimens was

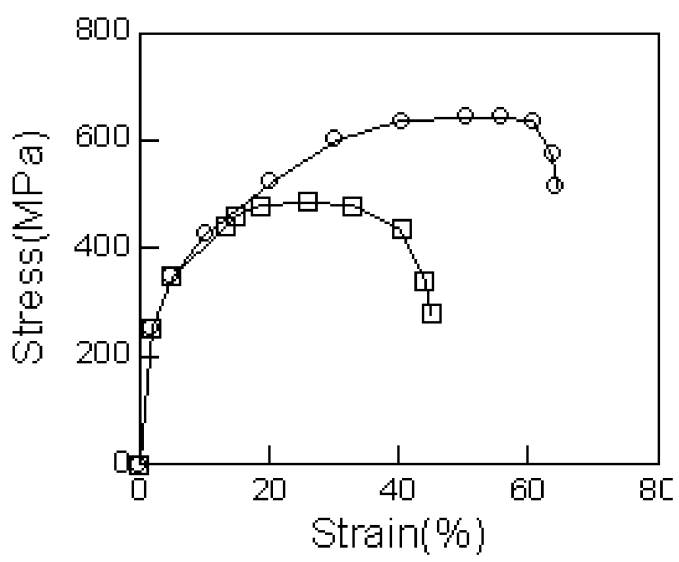

Figure 1. Stress-strain curves of 321 stainless steel in blank solution and the solution containing $0.005 \mathrm{~mol} / \mathrm{l} \mathrm{KI}$ ( $\square$, blank solution; $\mathrm{O}$, solution as in curve $\square+0.005 \mathrm{~mol} / \mathrm{l} \mathrm{KI}$ ). observed with $\mathrm{a} \times 30$ microscope. The strain distribution in front of the crack tip of the pre-cracked plate specimens in air, in the blank solution and in the solution with KI was measured by SPI (Suezou and Hiroyoshi 1985). For the electrochemical measurements, a saturated calomel electrode (SCE) was used as the reference electrode and all the values of the electrode potential are reported vs SCE. The polarization curves were measured on a PAR 332 electrochemical measuring system. All the tests were repeated three times to confirm the reliability of the results. The fractured surfaces of the failed specimens were observed by scanning electron microscope (SEM).

\section{Results}

\subsection{Stress strain curves}

Figure 1 shows plots of the stress vs strain of the steel in blank solution and in the solution containing KI. The results show that KI exerts significant inhibitive effects on the SCC of the system studied. The fracture strain in solutions with KI is significantly higher than that in blank solution.

\subsection{Effects of cross-head speeds on crack propagation rate}

Figure 2 shows crack rate-stress intensity factor $K$. It can be seen that the crack propagation rate decreased with the decrease of cross-head speeds, but when the cross-head speed was lower than $1 \times 10^{-5} \mathrm{~mm} / \mathrm{s}$, the plateau rate remained the same.

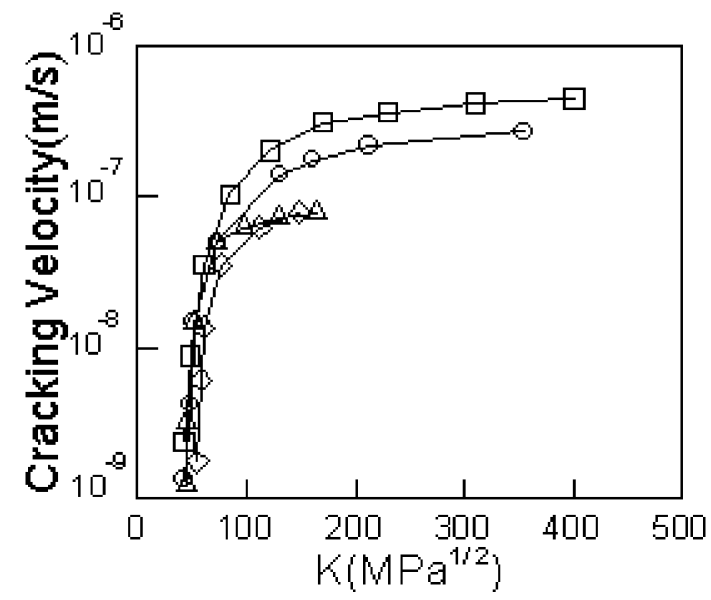

Figure 2. Crack propagation rate $\mathrm{d} a / \mathrm{d} t-K$ curves for 321 stainless steel in $0.5 \mathrm{~mol} / 1 \mathrm{HCl}+0.5 \mathrm{~mol} / \mathrm{l} \mathrm{NaCl}$ solution at different cross-head speeds $\left(\square, 5 \times 10^{-5} \mathrm{~mm} / \mathrm{s} ; \bigcirc, 2.5 \times 10^{-5} \mathrm{~mm} / \mathrm{s}\right.$; $\left.\Delta, 1 \times 10^{-5} \mathrm{~mm} / \mathrm{s} ; \diamond, 5 \times 10^{-6} \mathrm{~mm} / \mathrm{s}\right)$. 


\subsection{Effects of inhibitor KI on crack propagation}

As the crack rate remained the same at cross-head speeds of $1 \times 10^{-5} \mathrm{~m} / \mathrm{s}$ and lower, this was chosen to evaluate the effect of KI on crack propagation. Figure 3 shows the crack propagation rate-stress intensity factor $K$. When KI was added to the test solution, the crack propagation rate decreased.

\subsection{Polarization curves}

Polarization curves for AISI 321 stainless steel in blank solution and in the solution with KI are shown in figure 4 . It can be seen that when KI was added into the test solution, the anodic dissolution of steel was greatly reduced.

\subsection{Strain distribution in front of crack tip}

Figure 5 shows the strain $\left(\varepsilon_{\mathrm{xx}}\right)$ distribution in front of the crack tip of plate specimens loaded with $24 \mathrm{MPa}$ to $36 \mathrm{MPa}$ at a loading rate of $0.02 \mathrm{MPa} / \mathrm{s}$ in air, in the blank solution and in the solution with $0.005 \mathrm{~mol} / \mathrm{l} \mathrm{KI}$. The maximum strain and the area encircled by the contour with the same strain level in front of the crack tip in the blank solution are larger than those in air.

The deformation in front of the crack tip in the solution with KI is reduced compared with that in the blank solution, the maximum strain and the area encircled by the contour with the same strain level in front of the crack tip in the solution with $\mathrm{KI}$ are smaller than those in the blank solution.

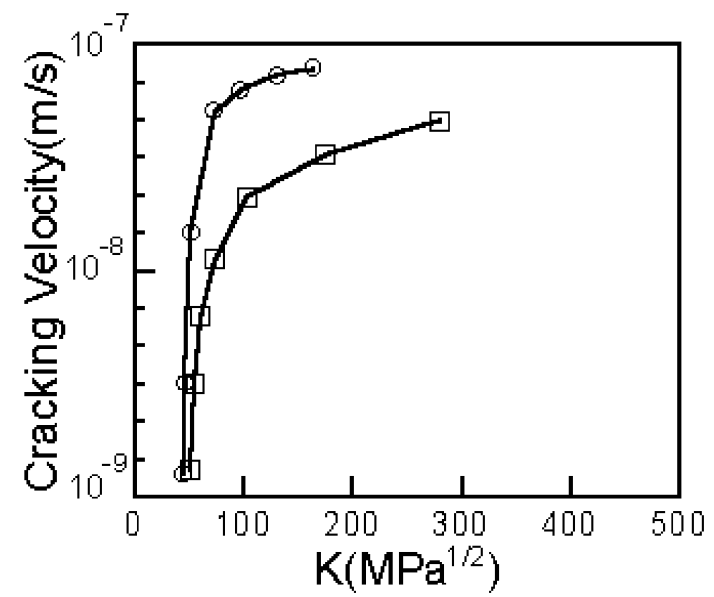

Figure 3. Crack velocity $\mathrm{d} a / \mathrm{d} t-K$ curves for single-edge precracked specimens in $0.5 \mathrm{~mol} / \mathrm{l} \mathrm{HCl}+0.5 \mathrm{~mol} / \mathrm{l} \mathrm{NaCl}$ solution and in the solution with $0.005 \mathrm{~mol} / \mathrm{l} \mathrm{KI}$ at cross-head speed of $1 \times 10^{-5} \mathrm{~mm} / \mathrm{s}(\mathrm{O}, 0.5 \mathrm{~mol} / 1 \mathrm{HCl}+0.5 \mathrm{~mol} / \mathrm{l} \mathrm{NaCl} ; \square$, as in $\mathrm{O}+0.005 \mathrm{~mol} / \mathrm{l} \mathrm{KI})$.

\subsection{Crack morphology}

The fractured surfaces of failed specimens were observed by scanning electron microscopy (SEM). A lot of cracks can be seen on the side surface of the rod specimen fractured in blank solution and the morphology of the fractured surfaces is typical of brittle cleavage. In the solution added with KI, a few cracks can be found on the side surface of the rod specimen and the fractured surface exhibits a lot of dimples that are typical of ductile fracture. Figure 6 shows the cracked surface morphology of the pre-cracked specimens in blank solution. The fractured surface is of brittle type at low $K$ stage. At high $K$ stage, the fractured surface is characterized as brittle cleavage with dimples in between. It means that the propagation of SCC cracks is discontinuous.

\section{Discussion}

Cao et al (1991) and Huang et al (1993) proposed a quantitative method for describing the effects of inhibitors on anodic and cathodic reactions and deduced equations as follows:

$$
\begin{aligned}
& I_{\mathrm{a}}^{\prime}=f_{\mathrm{a}} \cdot I_{\mathrm{a}}=f_{\mathrm{a}} \cdot I_{\text {corr }} \cdot \exp \frac{E-E_{\text {corr }}}{\beta_{\mathrm{c}}}, \\
& I_{\mathrm{c}}^{\prime}=f_{\mathrm{c}} \cdot I_{\mathrm{c}}=f_{\mathrm{c}} \cdot I_{\text {corr }} \cdot \exp \frac{E-E_{\text {corr }}}{\beta_{\mathrm{c}}},
\end{aligned}
$$

where $I_{\text {corr }}$ and $E_{\text {corr }}$ are respectively the corrosion current density and corrosion potential and $\beta_{\mathrm{a}}$ and $\beta_{\mathrm{c}}$ are the anodic and cathodic Tafel slopes in natural logarithmic scale in the uninhibited solution; $I_{\mathrm{a}}^{\prime}$ and $I_{\mathrm{c}}^{\prime}$ are the respective anodic and cathodic current densities in the solution with an additive and $I_{\mathrm{a}}$ and $I_{\mathrm{c}}$ are those in the uninhibited solution under the same electrode potential $\mathrm{E} ; f_{\mathrm{a}}$ and $f_{\mathrm{c}}$ are the corresponding action coefficients of the

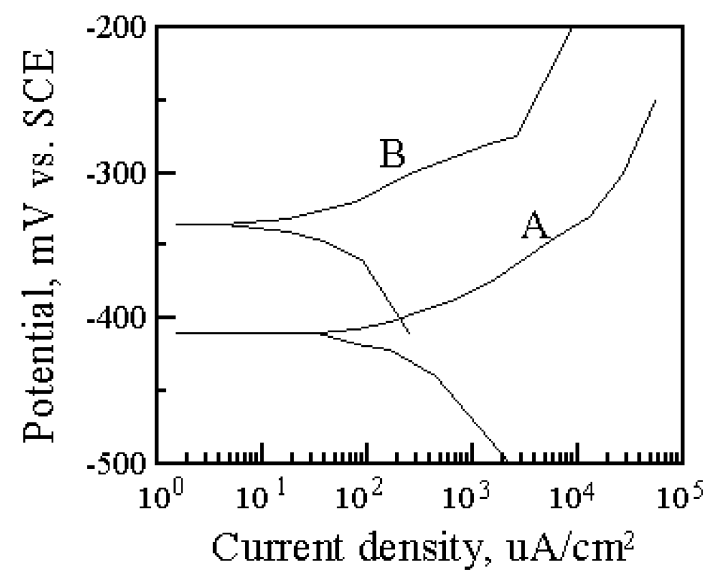

Figure 4. Polarization curves for AISI 321 stainless steel (A, $0.5 \mathrm{~mol} / 1 \mathrm{HCl}+0.5 \mathrm{~mol} / \mathrm{l} \mathrm{NaCl}$; $\mathrm{B}$, as in $\mathrm{A}+0.005 \mathrm{~mol} / \mathrm{l} \mathrm{KI}$ ). 

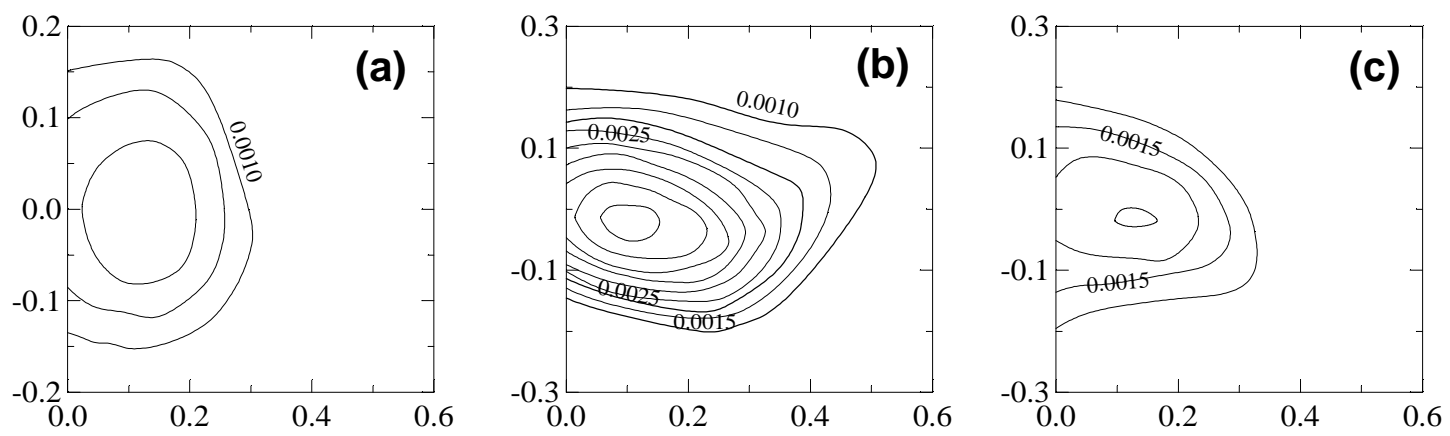

Figure 5. Strain distribution of the specimen in front of the crack tip in air (a), in the blank solution (b) and in the solution added with $0.005 \mathrm{~mol} / \mathrm{l} \mathrm{KI}(\mathbf{c})$. The crack tip is at $(0,0)$. The minimum strain is 0.001 and the step between each adjacent contour is $0 \cdot 0005$. The unit of the coordinates is in $\mathrm{mm}$.
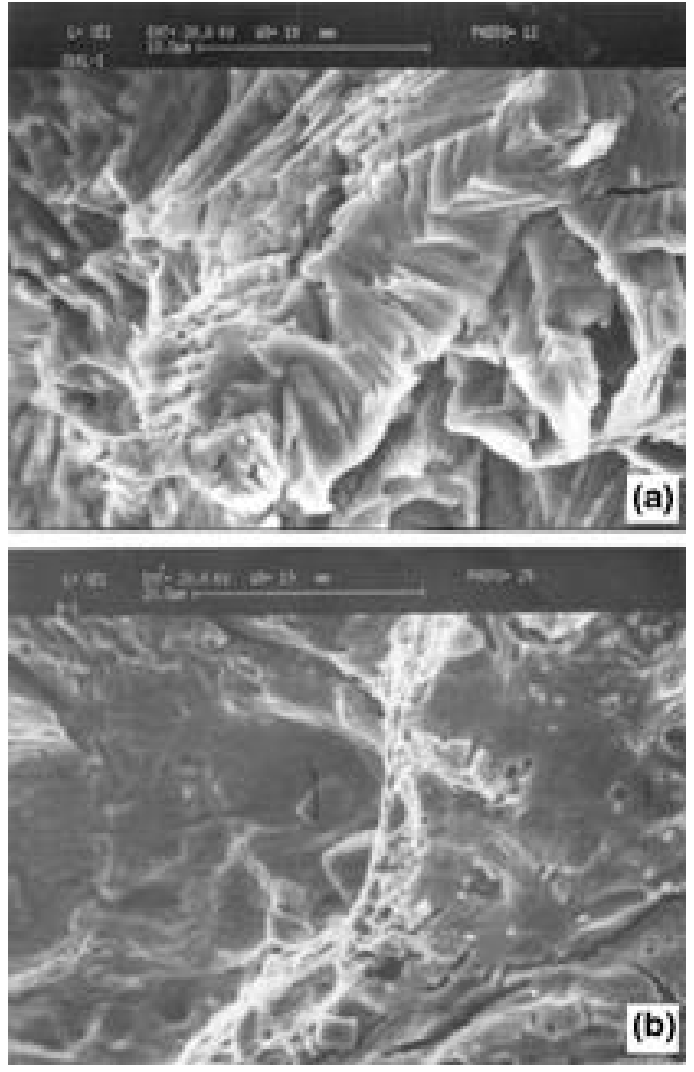

Figure 6. The cracked surface morphology of the pre-cracked specimens in blank solution. (a) At low $K$ stage and (b) at high $K$ stage.

additive on the anodic and cathodic reactions of the corrosion process.

The additive inhibits the corresponding reaction if the value of the corresponding coefficient is $<1$ and accelerates the reaction if it is $>1$. The Tafel slopes for anodic reaction in natural logarithmic scale estimated by a technique of curve-fitting in low polarization region ( $\mathrm{CaO}$ 1985) using the data shown in figure 4 are respectively 29.4 and $30 \cdot 1$ in the uninhibited and KI inhibited solutions. The corresponding cathodic reaction slopes are 48.8 and $52.3 \mathrm{mV}$ in these solutions. The corrosion current densities estimated for the two solutions are $0.51 \mathrm{~mA} / \mathrm{cm}^{2}$ and $0.064 \mathrm{~mA} / \mathrm{cm}^{2}$. The corrosion potentials measured in the two solutions are: $-370 \mathrm{mV}$ and $-303 \mathrm{mV}$. According to these data and (2), the calculated values of $f_{\mathrm{a}}$ and $f_{\mathrm{c}}$ are 0.009 and 0.34 for KI. The inhibition effect of $\mathrm{KI}$ on SCC is mainly due to the inhibition effect on the anodic reaction of the corrosion process.

Jones (1985) stated that anodic dissolution relieves strain hardening and reduces the fracture stress at the crack tip. The relief of strain hardening occurs by interaction of subsurface dislocations with divacancies generated by the anodic dissolution. The results presented here seem to confirm the hypothesis above. Since, if the mechanism proposed by Jones is correct, the deformation in front of the crack tip of the specimen in the blank solution would be easier than that in air and with the inhibitor added to the blank solution which has a strong inhibition effect on anodic reaction as discussed above, the deformation in front of the crack tip will be greatly reduced. The inference is verified by the experimental results shown in figure 5 . The strain hardening relief at the crack tip by anodic reaction did not induce any significant blunting of the crack tip and the fractured surface was characterized by brittle cleavage with no obvious evidence of electrochemical dissolution, this can be explained by the reduction in fracture stress at the crack tip induced by anodic reaction.

The fractured surface observation of pre-cracked specimens also supports the SCC mechanism presented by Jones (1985). The strain hardening relief and the reduction in fracture stress require the vacancies generated by anodic dissolution to diffuse into the crack tip to form divacancies to react with subsurface dislocations. At low $K$ values, the crack propagation velocity is very slow, the vacancies have sufficient time to diffuse into the crack tip and thus the fractured surface is characterized by brittle cleavage. At high $K$ values, the vacancies have little time to diffuse, so only a comparatively thinner brittle film is formed beyond the crack tip. After the 
brittle film fractured in high $K$ value, the stress at the crack tip was still high enough for ductile fracture to occur. The crack tip was blunted by ductile fracture and the crack velocity was lowered for a short period, then the metal at the crack tip reacted with the test solution to generate vacancies and the brittle fracture process repeated as discussed above. So the characteristic of the fractured surface is brittle cleavage with dimples in between.

\section{Conclusions}

(I) The SCC of AISI 321 stainless steel in acidic chloride solution is of active dissolution type and can be inhibited by the inhibitor KI. The inhibition effect of KI on the SCC is mainly due to the effect on the anodic reaction of the corrosion process.

(II) The results reported support the unified mechanism of SCC and CFC.

\section{Acknowledgement}

This work was financially supported by the Foundation of Chinese Educational Department for Overseas Student.

\section{References}

Cao Chu-Nan 1985 Chinese Society for Corrosion and Protection 5155 (in Chinese)

Cao Chu-Nan, Yan Qiangong, Lu Min and Lin Haichao 1991 Proc. 7th APCCC (Beijing: International Academic Publishers) pp 882-887

Chen Xujun and Wu Shanhe 1985 Proc. 6th Europ. symp. inh. (Ferrara: Elsevier Science Publishers) pp 677-689

Chinese Research Institute of Aeronautical Technology 1981 Handbook of stress intensity factor (Beijing: PRC Science Press) (in Chinese)

Collins J A and Monack M L 1973 Mater. Perf. 1211

Gnanamoorthy J B 1990 Mater. Perf. 2963

Huang Shuju 1988 Corrosion and protection of metals (China: Xi' an Jiaotong University Press) (in Chinese)

Huang Y L, Cao C N, Lu M and Lin H C 1993 Corrosion 49 644

Jones A 1985 Metall. Trans. A16 1133

Kain R M 1990 Mater. Perf. 2960

Newman R C and Procter R P M 1990 Br. Corros. J. 25 259

Oldfield J W and Todd B 1990 Mater. Perf. 2957

Suezou Nakadate and Hiroyoshi Saito 1985 Appl. Opt. 24 2172 